\title{
Correction to: Predictive factors of truncation artifacts in the arterial phase of Gd-EOB-DTPA-enhanced MRI: a nationwide multicenter study
}

\author{
Masakatsu Tsurusaki ${ }^{1}$ (D) Keitaro Sofue ${ }^{2} \cdot$ Hiromitsu Onishi $^{3} \cdot$ Satoshi Goshima ${ }^{4} \cdot$ Atsushi Higaki $^{5} \cdot$ Hiroyoshi Isoda $^{6}$. \\ Hiroki Haradome ${ }^{7} \cdot$ Kazunari Ishii $^{1} \cdot$ Takamichi Murakami $^{2}$
}

Published online: 4 February 2021

(c) Japan Radiological Society 2021

\section{Correction to: Japanese Journal of Radiology https://doi.org/10.1007/s11604-020-01052-x}

In the original publication of this paper, results section has been mistakenly published without consistency with Tables.

\section{Original:}

Results

Patients and overall analyses

Univariate analyses (Table 2) of patient factors revealed increased ORs for artifacts related to increased body weight (OR 1.025) and presence of cirrhosis (OR 1.605). In comparison, the ORs for TAs were found to be significantly lower for patients with hepatitis B (OR 0.485) and metastatic cancer (OR 0.416). Siemens and Toshiba equipment had significantly lower ORs for TAs than GE (OR 0.067, $P<0.001$ and OR $0.031, P<0.001$, respectively). In univariate analysis

The original article can be found online at https://doi.org/10.1007/ s11604-020-01052-x.

\section{Masakatsu Tsurusaki}

mtsuru@dk2.so-net.ne.jp

1 Department of Radiology, Faculty of Medicine, Kindai University, 377-2, Ohnohigashi, Osaka-sayama, Osaka 589-8511, Japan

2 Department of Radiology, Kobe University Graduate School of Medicine, Kobe, Hyogo, Japan

3 Department of Radiology, Osaka University Graduate School of Medicine, Suita, Osaka, Japan

4 Department of Radiology, Gifu University Graduate School of Medicine, Gifu, Gifu, Japan

5 Department of Radiology, Kawasaki Medical School, Kurashiki, Okayama, Japan

6 Department of Radiology, Kyoto University Graduate School of Medicine, Kyoto, Kyoto, Japan

7 Department of Radiology, Kitasato University School of Medicine, Sagamihara, Kanagawa, Japan
(Table 2) of imaging parameters, sequential k-space ordering (OR 3.245), shorter acquisition time ( $\leq 15 \mathrm{~s}$, OR 0.450), and chemical shift selective fat suppression (OR 0.298) were significant predictive factors of TA.

$\ldots$

GE equipment

Univariate analyses (Table 3) revealed an increased OR for TAs in patients with increased body weight (OR 1.025). In contrast, the ORs for TAs were significantly lower in patients with hepatitis B (OR 0.485) and metastatic cancer (OR 0.293).

\section{After the correction:}

Results

Patients and overall analyses

Univariate analyses (Table 2) of patient factors revealed increased ORs for artifacts related to increased body weight (OR 1.025) and presence of cirrhosis (OR 1.605). In comparison, the ORs for TAs were found to be significantly lower for patients with hepatitis B ( $\underline{\mathrm{OR} 0.406)}$ ) and metastatic cancer (OR 0.416). Siemens and Toshiba equipment had significantly lower ORs for TAs than GE (OR 0.067, $P<0.001$ and OR 0.031, $P<0.001$, respectively). In univariate analysis (Table 2 ) of imaging parameters, sequential k-space ordering ( $\mathrm{OR} 3.243$ ), shorter acquisition time $(\leq 15 \mathrm{~s}$, OR 0.251$)$, and chemical shift selective fat suppression (OR 0.298) were significant predictive factors of TA.

...

GE equipment

Univariate analyses (Table 3) revealed an increased OR for TAs in patients with increased body weight (OR 1.025). In contrast, the ORs for TAs were significantly lower in patients with hepatitis B (OR 0.485) and suspected metastatic cancer (OR 0.293).

Publisher's Note Springer Nature remains neutral with regard to jurisdictional claims in published maps and institutional affiliations. 